\title{
Equation-of-State Model for Temperature-Responsive Polymers with Tunable Response Onset
}

\author{
E. Manias* and Alexei M. Kisselev ${ }^{\dagger}$ \\ ${ }^{*}$ Dept. of Materials Science and Engineering, Pennsylvania State University, University Park, PA 16802, USA \\ ${ }^{\dagger}$ Dept. of Physics, Pennsylvania State University, University Park, PA 16802, USA
}

\begin{abstract}
The phase behavior of aqueous solutions of temperature-responsive (ethylene oxide)/ethylene copolymers with tunable lower critical solution temperatures (LCST) is explored using an equation of state approach. The LCST in water of these polymers is tailored by their chemical composition, specifically by the balance of hydrophilic to hydrophobic groups in the polymer. The general formalism of the lattice-fluid with hydrogen-bonding theory, adjusted here to account for multiple types of hydrogen bonds, is employed, and the theoretical predictions are compared with experimental systems. The developed theoretical model is shown to be effective in describing the phase behavior of these systems, and the model parameters seem to be transferable between different homologous copolymer series.
\end{abstract}

Keywords: Equation-of-State Theory; Smart Polymers; Temperature-Responsive Polymers.

PACS: $82.60 . \mathrm{Lf} ; 83.80 . \mathrm{Rs} ; 82.35 . \mathrm{Jk}$

\section{INTRODUCTION}

Aqueous polymer solutions are abundant in nature and also important from a technological viewpoint, but, at the same time, represent systems whose theoretical description is rather challenging. These challenges arise from the fact that systems of molecules interacting with strong specific hydrogen-bonding interactions deviate remarkably from normal solution behavior $[1,2,3,4]$. One such a deviation is the existence of a lower critical solution temperature (LCST), above which the polymer becomes insoluble in water. Poly(ethylene oxide) (PEO) is probably the most investigated water-soluble synthetic polymer with an LCST, including considerable theoretical attention in recent years for water solutions [5, 6, e.g.] and in $1 \mathrm{~nm}$ confinements [7, 8, 9, e.g.]. Its LCST, associated with its hydrogen-bonding to water, occurs at $\mathrm{T} \geq 100^{\circ} \mathrm{C}$, i.e., above the boiling point of water, thus limiting its use for applications requiring a temperatureresponse. However, it has been shown that the LCST of water-soluble polymer can be decreased by proper addition of hydrophobic segments to the polymer [10,11], as long as hydrophilic and hydrophobic segments are not lumped together in extended blocks (such a blockiness would facilitates micellar type of aggregates/collapse [12,13] rather than a 'normal' coil-to-globule transition [14]).

In this work ${ }^{1}$, we will focus on this last type of oligo-ethylene-oxide/oligo-ethylene linear alternating copolymers, which exhibit LCST transitions tailored at varied temperatures (from 7 to $80{ }^{\circ} \mathrm{C}$ ) via the copolymer composition [11]. Specifically, focusing on these polymers, we describe their phase behavior by an established equation of state theoretical framework, the lattice-fluid with hydrogen-bonding (LFHB) [4].

\section{THEORETICAL MODEL AND RESULTS}

From an Equation-of-State theoretical viewpoint, one can treat van der Waals (physical) interactions with the wellestablished Sanchez-Lacombe compressible lattice-fluid (LF) model [1, 2], combined with the chemical association approach [3] to account for hydrogen-bonding (chemical interactions). Such a model was proposed by Panayiotou and Sanchez -known as the lattice-fluid with hydrogen-bonding (LFHB) theory [4]- where the chemical (hydrogenbonding) contributions are treated through an enumeration of pair interactions between various hydrogen-bonding donor and acceptor groups. Namely, the chemical contributions of LFHB are based on the combinatorial expression for the number of ways of forming hydrogen bonds. The basic approximation of the model is that physical (van der

\footnotetext{
${ }^{1}$ Acknowledgements. Support provided by ONR (grant\# 00014-05-1-0614) and NSF (DMR-0602877, an MPS/Polymers and MWN award). 
Waals) and chemical (hydrogen-bonding) forces are effectively decoupled, i.e., the canonical partition function can be factored as $Q=Q_{L F} Q_{H B}$ so that $Q_{L F}$ disregards the existence of hydrogen bonds and considers only physical intermolecular interactions, while $Q_{H B}$ accounts only the hydrogen bonding.

Nomenclature: We define our system to contain $N_{k}$ molecules of $k$-th type, at temperature $T$ and external pressure $P$. There are $m$ types of proton-donor groups and $n$ types of proton-acceptor groups, with $d_{i}{ }^{k}$ being the number of donor groups of $i$-th type in each molecule of $k$-th type and, equivalently, $a_{j}{ }^{k}$ number of $j$-th type acceptor groups in each $k$-th type molecule.

Lattice-Fluid. For the lattice-fluid theory, molecules are arranged on a quasi-lattice of $N_{r}$ sites, $N_{0}$ of which are empty. Each molecule of $k$-th type is divided into $r_{k}$ segments of close-packed volume $v_{k}^{*}$ in the pure state and average (mean-field) interaction energy $\varepsilon_{k}^{*}$. The following combining and mixing rules are assumed

$$
v^{*}=\sum_{k=1}^{t} \phi_{k} v_{k}^{*} \text { and } \varepsilon^{*}=\frac{1}{2}\left(\sum_{p=1}^{t} \phi_{p} s_{p}\right)\left(\sum_{k=1}^{t} \sum_{l=1}^{t} \theta_{k} \theta_{l} \varepsilon_{k l}\right), \text { with } \varepsilon_{k l}=2 \xi_{k l}\left(\frac{\varepsilon_{k}^{*} \varepsilon_{l}^{*}}{s_{k} s_{l}}\right)^{1 / 2}
$$

where $x_{k}$ are the mole fractions, $\phi_{k}$ are the segment fractions (defined as $\phi_{k}=x_{k} r_{k} / r$ ), and $\theta_{k}$ are the surface fractions (defined as $\theta_{k}=\phi_{k} s_{k} / \sum_{l=1}^{t} \phi_{l} s_{l}$, where $s_{k}$ is the average number of contacts per $k$-th segment, equivalent to a surface to volume ratio of that segment). A Berthelot-type combining rule is adopted for $\varepsilon_{k l}$ ( $\xi_{k l}$ is a dimensionless parameter, expected to have values close to unity). The total lattice-fluid volume of the system is given by $V_{L F}=N_{r} v^{*}=r N v^{*} \tilde{v}$, where the reduced volume $(\tilde{v}=1 / \tilde{\rho})$ is defined of the basis of the reduced density $\left(\tilde{\rho}=r N / N_{r}\right)$. Similarly, the total potential energy of the system, as derived in $[2,4]$ taking into account only nearest-neighbor interactions and ignoring interactions with empty sites, is given by $-E_{L F}=r N \tilde{\rho} \varepsilon^{*}$.

Hydrogen-Bonding. The interaction energies due to hydrogen-bonding contribution are in excess of the physical interactions, and is denoted $E_{i j}{ }^{0}$ for the hydrogen bond between a donor of the $i$-th type and an acceptor of the $j$-th type. If $S_{i j}{ }^{0}$ is the entropy loss associated with the $(i, j)$ bond formation, $V_{i j}{ }^{0}$ is the respective volume change, and there exist $N_{i j}$ bonds of the $(i, j)$ type each with $P_{i j}$ probability of formation, the number of ways of distributing the $N_{i j}$ bonds among the functional groups of the system is $\Omega$ and the total hydrogen-bonding energy of the system is $E_{H B}$ :

$$
E_{H B}=\sum_{i=1}^{m} \sum_{j=1}^{n} N_{i j} E_{i j}^{0} \text { and } \Omega=\prod_{i=1}^{m} \frac{N_{d}{ }^{i} !}{N_{i 0} !} \prod_{j=1}^{n} \frac{N_{a}{ }^{j} !}{N_{0 j} !} \prod_{i}^{m} \prod_{j}^{n} \frac{P_{i j} N_{i j}}{N_{i j} !}, \text { with } P_{i j}=\frac{\tilde{\rho}}{r N} \exp \left[\frac{S_{i j}{ }^{0}}{R}\right]
$$

where $N_{d}{ }^{i}$ and $N_{a}{ }^{j}$ are the total number of $i$-type donor groups and $j$-type acceptor groups, leaving $N_{i 0}$ and $N_{0 j}$ number of unbonded $i$-donors and $j$-acceptors.

Free energy and equations of state. The respective Gibbs free energy in the above framework, i.e., Lattice Fluid Theory with Hydrogen-Bonding (LFHB) [4], consists of two terms $\left(G=G_{L F}+G_{H B}\right)$, with the lattice-fluid term given by

$$
\frac{G_{L F}}{k_{B} T}=r N\left\{-\frac{\tilde{\rho}}{\tilde{T}}+\frac{\tilde{P} \tilde{v}}{\tilde{T}}+(\tilde{v}-1) \ln (1-\tilde{\rho})+\frac{1}{r} \ln \tilde{\rho}+\sum_{k=1}^{t} \frac{\phi_{k}}{r_{k}} \ln \frac{\phi_{k}}{\omega_{k}}\right\}
$$

and the hydrogen-bonding contribution is given by

$$
\frac{G_{H B}}{k_{B} T}=r N\left\{\sum_{i=1}^{m} \sum_{j=1}^{n} v_{i j}+\sum_{i=1}^{m} v_{d}{ }^{i} \ln \frac{v_{i 0}}{v_{d}{ }^{i}}+\sum_{j=1}^{n} v_{a}{ }^{j} \ln \frac{v_{0 j}}{v_{a}{ }^{j}}\right\}
$$

where the system volume is $\left.V=r N \tilde{v} v^{*}+\sum_{i=1}^{m} \sum_{j=1}^{n} N_{i j} V_{i j}{ }^{0}\right)$, the reduced pressure is $\tilde{P}=P v^{*} / \varepsilon^{*}$, and the reduced temperature is $\tilde{T}=R T / \varepsilon^{*}$; various H-bonding fractions are $v_{i j}=N_{i j} / r N, v_{i 0}=N_{i 0} / r N, v_{0 j}=N_{0 j} / r N, v_{d}{ }^{i}=N_{d}{ }^{i} / r N$, and $v_{a}{ }^{j}=N_{a}{ }^{j} / r N ; \omega_{k}$ is the number of configurations available to a $r_{k}$-mer in the close-packed pure state, $\omega_{k}$ is treated as a constant and will cancel out in all applications of interest here (for more details see e.g. [4]). The minimization conditions with respect to $\tilde{v}$ and $N_{i j}$ (i.e., $\partial G / \partial \tilde{v}=0$ and $\partial G / \partial N_{i j}=0$ ), provide the equation of state for the reduced density and a system of equations for the fractions of hydrogen bonds in the system, respectively:

$$
\tilde{\rho}^{2}+\tilde{P}+\tilde{T}\left[\ln (1-\tilde{\rho})+\tilde{\rho}\left(1-\frac{1}{\bar{r}}\right)\right]=0 \quad \text { and } \quad \frac{v_{i j}}{v_{i 0} v_{0 j}}=\tilde{\rho} \exp \left[\frac{-G_{i j}{ }^{0}}{R T}\right]
$$

where $G_{i j}{ }^{0}=E_{i j}{ }^{0}+P V_{i j}{ }^{0}-T S_{i j}{ }^{0}$. The reduced density equation of state together with the H-bond fractions equations (5) can serve as a system of equations of state. The chemical potential of the $k$-th component is the sum of the partial derivatives with respect to $N_{k}$ of the two free energy terms (eq. 3 and 4). 
Binary mixture. Focusing on the phase behavior of a polymer-solvent binary mixture (index 1 denotes solvent, and index 2 polymer, with mole fractions $x_{i}$, and corresponding segment fractions $\phi_{i}$ and surface fractions $\theta_{i}$ ), then simply $x_{2}=1-x_{1}=N_{2} /\left(N_{1}+N_{2}\right)$ and $\phi_{2}=1-\phi_{1}=r_{2} x_{2} / r$, with $\theta_{2}=\phi_{2} /\left(\phi_{2}+\phi_{1} s_{1} / s_{2}\right)$, where $s_{1} / s_{2}$ is ratio of the surface area per unit characteristic volume for solvent and polymer. The mixing and combining rules simplify to

$$
v^{*}=\phi_{1} v_{1}^{*}+\phi_{2} v_{2}^{*} \text { and } \varepsilon^{*}=\phi_{1} \varepsilon_{1}^{*}+\phi_{2} \varepsilon_{2}^{*}-\phi_{1} \theta_{2} R T X_{12},
$$

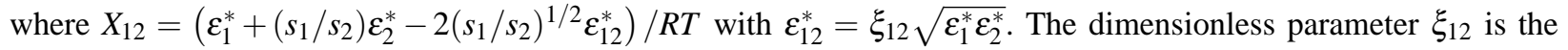
only free parameter of the model, and is expected to have values close to unity.

Aqueous solutions of PEO and of P(EO-alt-EE) copolymers. Application of this LFHB theory to the water-PEO solution phase behavior can yield the $\xi_{12}$ parameter for EO. For this system, there are two types of H-bonds, waterwater and water-polymer, each water molecule has 2 donors and 2 acceptors, and each PEO molecule has $a$ acceptors, where $a$ is the number of ether oxygens in each PEO. There will be three equations of state, one for the reduced density and one for each H-bond type (eq. 5). LF and HB parameters of the model were obtained from literature (fig. 1, $[4,15,16]$ ) and the ratio of surface areas per unit characteristic volume was calculated based on hard-sphere parameters $\left(s_{1} / s_{2}\right)^{E O}=1.3424$. The dimensionless variable $\xi_{12}$ is treated as a fitted free parameter, and for the phase diagram of $M_{\mathrm{w}}=10^{5} \mathrm{PEO}$ [17] the LFHB fit yields $\xi_{12}{ }^{E O}=1.0472$ (fig. 1). This value of $\xi_{12}{ }^{E O}$ is used in all later calculations of the phase diagrams of our "polyester" and "polyamide" ethylene oxide copolymers without any further change. The LFHB model can be extended to describe the phase behavior of the $\left[(E O)_{m}-(E E)_{n}\right]$ copolymers, by introducing only one more parameter for the EE monomer $\left(\xi_{12}{ }^{E E}\right)$ which can be obtained by fitting only one of the copolymer binodals to the LFHB model (fig. 1, fit of an $m=13, n=6$ ester copolymer [11] yields $\xi_{12}{ }^{E E}=1.0537$ ). All the rest of the copolymers will now be described by these same parameters, while accounting for the donor/acceptor groups of the ester and amide linkage groups, and for the variation in EO/EE copolymer composition via $m$ and $n$ by using the following simple combination rule (the weighted average):

$$
\xi_{12}=\frac{m}{m+n} \xi_{12}^{E O}+\frac{n}{m+n} \xi_{12}^{E E} \text { and } \quad \frac{s_{1}}{s_{2}}=\frac{m}{m+n}\left(\frac{s_{1}}{s_{2}}\right)^{E O}+\frac{n}{m+n}\left(\frac{s_{1}}{s_{2}}\right)^{E E}
$$

Based on the hard-sphere model $\left(s_{1} / s_{2}\right)^{E E}=1.3266$. Replacement of EO segments with EE segments also decreases the number of hydrogen bonding sites per polymer molecule to $a_{2}^{2}=\left(a_{2}{ }^{2}\right)^{P E O} \cdot m /(m+n)$, where $\left(a_{2}{ }^{2}\right)^{P E O}=$ $M_{\mathrm{w}}^{\text {poly }} / M_{\mathrm{w}}^{E O}$.

This approach provides excellent agreement with the experiments (fig. 2), which strongly implies that the copolymer phase behavior is similar in nature as the aqueous phase behavior of PEO only temperature-shifted by the hydrophobic EE group contributions, and the LFHB parameters are transferable between homologous polymers. The present model

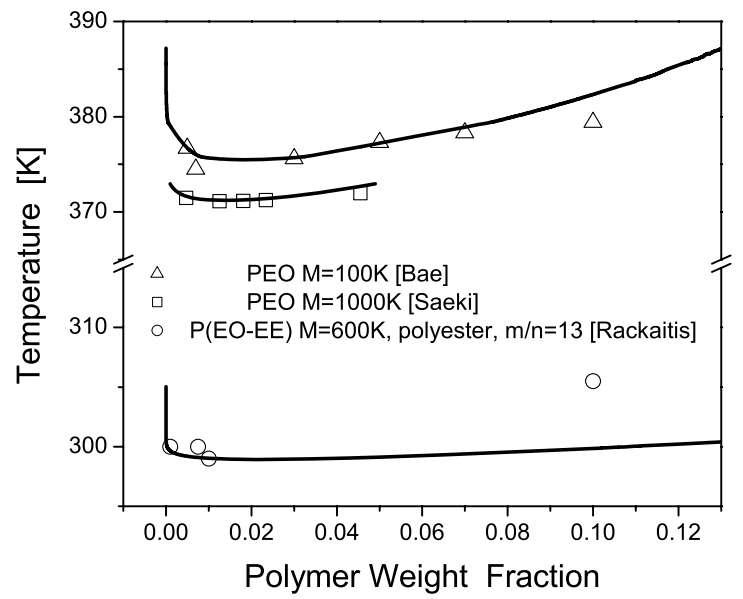

\begin{tabular}{lccc}
\hline $\begin{array}{l}\text { Lattice fluid } \\
\text { parameters }\end{array}$ & $\begin{array}{c}T^{*} \\
(\mathrm{~K})\end{array}$ & $\begin{array}{c}P^{*} \\
(\mathrm{~Pa})\end{array}$ & $\begin{array}{c}\rho^{*} \\
\left(\mathrm{~kg} / \mathrm{m}^{3}\right)\end{array}$ \\
\hline $\mathrm{H}_{2} \mathrm{O}$ & 518 & $4.75 \cdot 10^{8}$ & 853 \\
$\mathrm{PEO}$ & 541 & $6.05 \cdot 10^{8}$ & 1172 \\
\hline $\mathrm{H}-$ bonding & $E_{i j}^{0}$ & $S_{i j}{ }^{0}$ & $V_{i j}{ }^{0}$ \\
parameters & $(\mathrm{J} / \mathrm{mol})$ & $(\mathrm{J} / \mathrm{mol} \cdot \mathrm{K})$ & $\left(\mathrm{m}^{3} / \mathrm{mol}\right)$ \\
\hline$-\mathrm{OH} \cdots-\mathrm{OH}$ & $-1.55 \cdot 10^{4}$ & -16.6 & $-4.2 \cdot 10^{-6}$ \\
$-\mathrm{OH} \cdots-\mathrm{O}-$ & $-1.42 \cdot 10^{4}$ & -16.0 & $-8.5 \cdot 10^{-7}$ \\
$-\mathrm{OH} \cdots \mathrm{C}=\mathrm{O}$ & $-1.60 \cdot 10^{4}$ & -15.8 & $-8.5 \cdot 10^{-7}$ \\
$\mathrm{~N}-\mathrm{H} \cdots-\mathrm{OH}$ & $-1.25 \cdot 10^{4}$ & -7.8 & $-8.5 \cdot 10^{-7}$ \\
\hline
\end{tabular}

FIGURE 1. (left) Fitting of the model on experiments: fitting to PEO/water solutions[17, 18] yields the single adjustable parameter for EO monomer, $\xi_{12}^{\mathrm{EO}}$; subsequent fitting to one ester copolymer binodal yields $\xi_{12}^{\mathrm{EE}}$ for the EE monomer; both $\xi_{12}^{\mathrm{EO}}$ and $\xi_{12}^{\mathrm{EE}}$ are then used for all other ester and amide copolymers with no further adjustment. (right) The rest (unadjustable) LFHB parameters. 

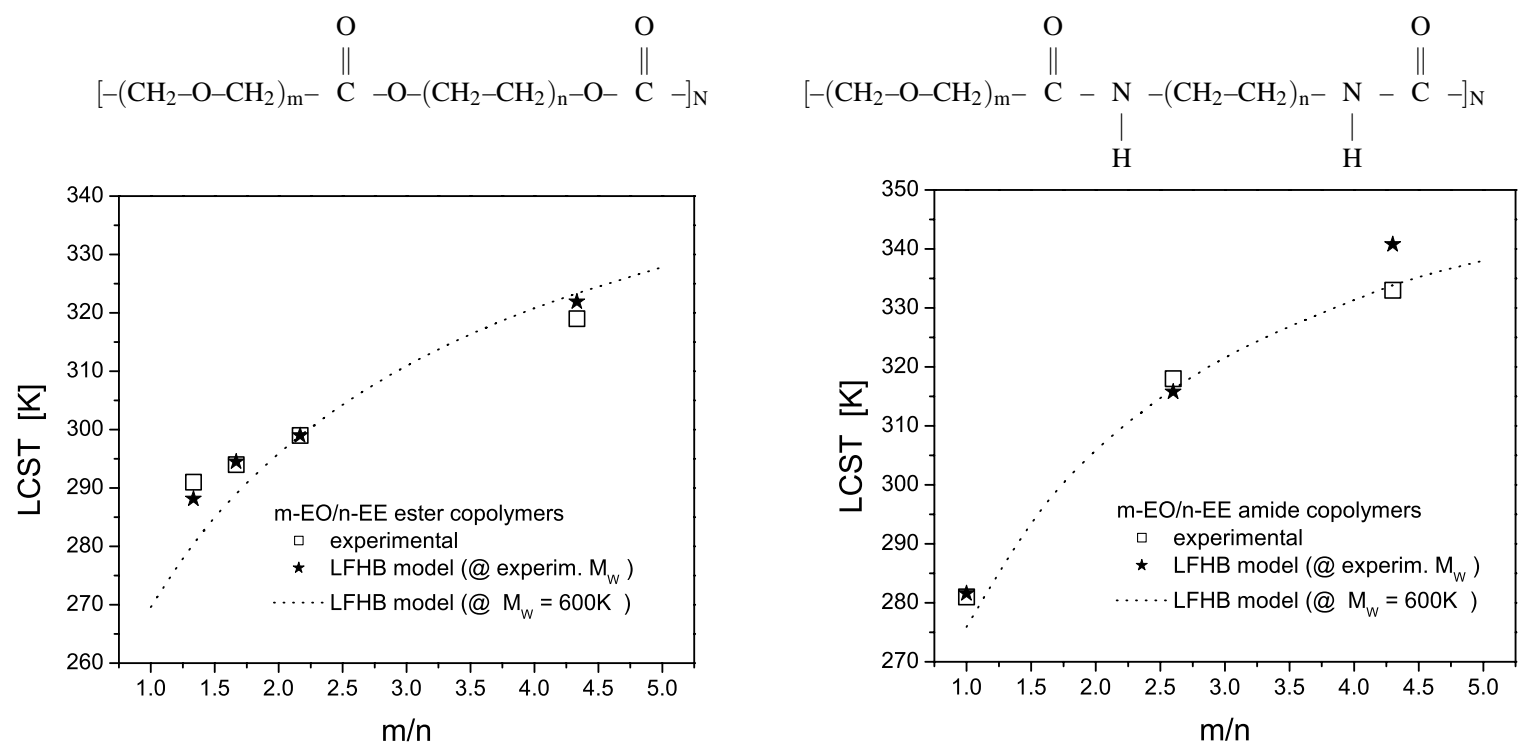

FIGURE 2. LCST dependence on the hydrophilic/hydrophobic balance $m / n$ for $\mathrm{m}$-EO/n-EE polyester (left) and polyamide (right) copolymers. For the experimental $m / n$ polymers the LCST with explicit $M_{\mathrm{W}}$ dependence (symbols) are calculated, whereas the LCST for $M_{\mathrm{W}}=600 \mathrm{~K}$ is also provided for a wide $m / n$ range (line).

takes into account both water-polymer and water-water H-bonding, however it does not account for the orientation of donor and acceptor sites with respect to each other, due to the purely statistical nature of the model. Thus, the model retains a mean-field character and inherent drawbacks, which makes it impossible to account for difference between alternating, random, and especially highly-segmented or block copolymers, or account for end-group effects (all of which are extremely important for the phase behavior of the copolymers in question).

Higher fidelity mean-field approaches, including those based on a quasi-chemical lattice-fluid framework and on hydrogen bonding cooperativity, as well as molecular based simulations, should be applied, if one is to obtain more insights into the phase behavior -and ultimately into quantitative design principles- of polymers with tunable temperature response.

\section{REFERENCES}

1. I. C. Sanchez, and R. H. Lacombe, Macromolecules 11, 1145-1156 (1978).

2. C. G. Panayiotou, Macromolecules 20, 861-871 (1987).

3. C. Panayiotou, and I. C. Sanchez, Macromolecules 24, 6231-6237 (1991).

4. C. Panayiotou, and I. C. Sanchez, J. Phys. Chem. 95, 10090-1097 (1991).

5. S. Bekiranov, R. Bruinsma, and P. Pincus, Phys. Rev. E 55, 577-585 (1997).

6. E. E. Dormidontova, Macromolecules 35, 987-1001 (2002).

7. V. Kuppa, S. Menakanit, R. Krishnamoorti, and E. Manias, J. Polym. Sci. B: Polym. Phys. 41, 3285-3298 (2003).

8. V. Kuppa, and E. Manias, J. Chem. Phys. 118, 3421-3429 (2003).

9. E. Manias, V. Kuppa, D. K. Yang, and D. B. Zax, Colloids Surf., A 187, 509-521 (2001).

10. E. Manias, M. Rackaitis, and K. E. Strawhecker, US Patent 7,011,930 (2006).

11. M. Rackaitis, K. Strawhecker, and E. Manias, J. Polym. Sci. B: Polym. Phys. 40, 2339-2342 (2002).

12. G. Bokias, D. Hourdet, and I. Iliopoulos, Macromolecules 33, 2929-2935 (2000).

13. J. Virtanen, S. Holappa, H. Lemmetyinen, and H. Tenhu, Macromolecules 35, 4763-4769 (2002).

14. A. N. Rissanou, S. H. Anastasiadis, and I. A. Bitsanis, J. Polym. Sci. B: Polym. Phys. 44, 3651-3666 (2006).

15. R. B. Gupta, C. G. Panayiotou, I. C. Sanchez, and K. P. Johnston, AIChE J. 38, 1243-1253 (1992).

16. A. K. Lele, M. V. Badiger, M. M. Hirve, and R. A. Mashelkar, Chem. Eng. Sci. 50, 3535-3545 (1995).

17. Y. C. Bae, J. J. Shim, D. S. Soane, and J. Prausnitz, J. Appl. Polym. Sci. 47, 1193-1206 (1993).

18. S. Saeki, N. Kuwahara, M. Nakata, and M. Kaneko, Polymer 17, 685-689 (1976). 